\title{
X-ray diffraction investigation of a spin crossover hysteresis loop.
}

\author{
P Guionneau ${ }^{1, *}$, F Le Gac ${ }^{1}$, S Lakhoufi ${ }^{1}$, A Kaiba ${ }^{1}$, J-F Létard ${ }^{1}$,P Négrier $^{2}$, D \\ Mondieig $^{2}$, J A K Howard ${ }^{3}$, J-M Léger ${ }^{4}$
}

${ }^{1}$ Institut de Chimie de la Matière Condensée de Bordeaux, ICMCB-CNRS, Université Bordeaux I, 87 Av. dr A. Schweitzer, 33608 Pessac - France.

${ }^{2}$ Centre de Physique Moléculaire Optique et Hertzienne, UMR Université Bordeaux I CNRS 5798, 33405 Talence Cedex, France

${ }^{3}$ Chemical Crystallography Group, Chemistry Department, Durham University, South Road, Durham DH1 3LE, United Kingdom

${ }^{4}$ Laboratoire de Pharmacochimie, Université Victor Segalen Bordeaux II, 146 rue Léo Saignat, 33076 Bordeaux, France

\section{*E-mail: guio@icmcb-bordeaux.cnrs.fr}

The nature and the mechanism of the magnetic hysteresis for the thermal spin crossover exhibited by an iron(II) compound is investigated by mean of variable temperature powder and single crystals X-ray diffraction. The unit cell temperature dependence clearly evidences the amplitude of the strong structural re-arrangement that accompanied the spin crossover - corresponding to a variation of $8.6 \%$ for a unit cell parameter - as well as the structural hysteresis width. To this regard, the present $\mathrm{X}$-ray study reveals significant differences in the spin crossover features according to the nature of the sample - powder or single crystal - that should be taken into account in the physical properties analysis. Concerning the interplay between structural and magnetic transitions, quenching effects show that the structural transition and the spin crossover are indissociable. Furthermore, investigations of the mechanism itself of the thermal spin crossover confirm the presence of spin-like domains in the conversion region, either in the cooling or in the warming loops. The non-dependence with temperature of these domains inside the hysteresis loop demonstrates the stability of the microscopic and macroscopic structures in the corresponding thermodynamic conditions. This result is of interest in the context of the potential use of hysteresis loops to obtain high temperature photo-conversion. 


\section{Introduction}

The solid state reversible modification of the electronic configuration in transition ion from a high spin (HS) state to a low spin (LS) state, namely the spin crossover, is increasingly studied either for fundamental interests or potential applications [1]. The spin crossover that can be driven by a variation of thermodynamic conditions or by an electromagnetic radiation shows a so large richness of magnetic, optical and structural features that numerous points are to explore further, going, for example, from the optimization of the characteristic temperatures to fundamental unexplained points, such as the mechanism of the spin crossover propagation through the material.

In regard to the mechanism of the spin crossover phenomenon, one of the crucial point to elucidate yet concerns the distribution of the entities that adopt the same spin state through the macroscopic sample when the conversion is not complete, as for example at $T_{1 / 2}$ where half of the entities are in the HS state and half in the LS state. Three situations could be envisaged a priori: (1) a random repartition corresponding to an homogeneous distribution at the sample scale, (2) a spin-like domain repartition where all the entities within a same domain adopt the same spin state or (3) the occurrence of an intermediate structural phase where HS and LS entities are structurally ordered. These situations can be easily distinguished experimentally through variable temperature X-ray diffraction: (1) gives Bragg peaks reflecting an average of the microscopic distribution and the position of the peaks continuously changes with temperature, (2) gives a split of each Bragg peak in a HS and a LS component such as the position of these peaks barely changes with temperature while the intensities increase or decrease proportionally to the macroscopic spin conversion level and (3) corresponds to significant change in the reciprocal space as at least two entities must be structurally independent.

Despite early preliminary attempts [2], it is only very recently that accurate X-ray diffraction experiments were conducted to investigate the mechanism of transition. Spin domains, (2), have been observed either for the thermal or the photo-induced spin crossover in a few materials [3-6] and examples of continuous process, (1), [7] and of intermediate structural phase, (3), [8] have been reported. More intricate phenomena like incommensurate moduling have also been observed [9].

Moreover, one recurrent question mark about the mechanism of the spin crossover phenomenon concerns the links between structural and spin transitions. Is the former drives the latter or vice versa?

Within this context, we investigated the mechanism along and inside an hysteresis loop as well as the synergy between the spin transition and the structural transition in the mononuclear iron(II) complex showing one of the largest magnetic hysteresis loop, namely [Fe(PM-PeA $\left.)_{2}(\mathrm{NCS})_{2}\right][10]$. 


\section{Experimental}

\section{1. $\mathrm{X}$-ray powder diffractograms in the range $[100-300 \mathrm{~K}]$}

In order to cover the hysteresis loop, X-ray diffractograms were recorded every $10 \mathrm{~K}$ when cooling from 280 to 120 and then when warming up from 120 to 280 with additional temperature around $\mathrm{T}_{1 / 2}$. Moreover, diffractograms were registered every $5 \mathrm{~K}$ by warming directly from $188 \mathrm{~K}$, $\mathrm{T} 1 / 2 \downarrow$, to $238 \mathrm{~K}, \mathrm{~T} 1 / 2 \uparrow$, to investigate inside the hysteresis loop. X-ray high-resolution diffraction patterns were recorded by means of a horizontally mounted INEL cylindrical position sensitive detector (CPS 120) by gas ionisation (Argon $+\mathrm{C}_{2} \mathrm{H}_{6}$ ) using Debye-Scherrer geometry (angular step ca. $0.029^{\circ}-2 \theta$ ) [11]. Monochromatic $\mathrm{CuK \alpha} \alpha_{1}$ radiation was selected as incident beam. Low temperature measurements were achieved with an Oxford Cryosystems $\mathrm{N}_{2}$ cryostream which provide isothermal experiments at different temperatures. The generator power was set to $40 \mathrm{kV}$ and $25 \mathrm{~mA}$. Samples were introduced in $0.5 \mathrm{~mm}$ diameter Lindemann glass capillaries, the latter rotating around its axis during the experiment in order to minimize preferential orientations of the crystallites. The acquisition time was set to an hour in order to obtain reflections with exploitable intensities. External calibration using $\mathrm{Na}_{2} \mathrm{Ca}_{3} \mathrm{Al}_{2} \mathrm{~F}_{14}$ [12] cubic phase combined with a low-angle calibration using silver behenate [13] was performed by means of cubic spline fittings.

\subsection{Accurate unit cell from single crystal experiments in the range [140 - $280 \mathrm{~K}$ ]}

Most of the single crystals explode at the spin transition due to the strong structural modifications. Very small prismatic single crystals of approximate dimensions of $10 \mu \mathrm{m}$ were consequently used because they are less affected by such deterioration. Moreover, the crystals were coated in an oil in the expectation of limiting the damages induced by the transition. In order to get enough diffraction peaks, the temperature dependence of the unit cell was investigated using a Rigaku Rapid R-axis diffractometer using a MM007 microfocus rotating anode generator with $\mathrm{Cu}$ radiation.

\subsection{Quenching single crystal experiments}

Single crystals were flash cooled to $30 \mathrm{~K}$ and investigated using an Oxford Cryosystems Helix open flow He gas cryostat combined with a Bruker SMART-CCD area detector diffractometer using the Mo K $\alpha$ radiation. First a full data collection was performed at $30 \mathrm{~K}$. The diffraction frames were integrated using the SAINT package. The structural determination by direct methods and the refinement of the atomic parameters based on full-matrix least squares on $\mathrm{F}^{2}$ were performed using the SHELX-97 [14] programs within the WINGX package [15]. Crystal data: $30 \mathrm{~K}, \mathrm{a}=15.681(1) \AA$,

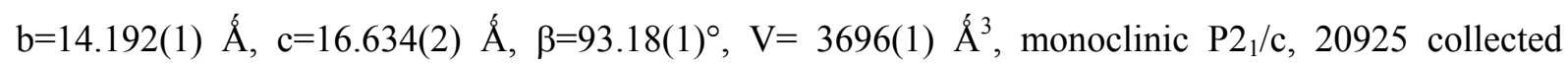
reflections for 7916 independent $(\mathrm{Rint}=0.04), \mathrm{R}=0.047$, wR2 $=0.109$. Full experimental and crystal 
data can be obtained with the supplementary crystallographic data for this paper, number CCDC632777 .

After data collection at $30 \mathrm{~K}$, unit cell parameters were determined by warming the sample. The experiments were reproduced. All investigated samples show no modification of the unit cell from 30 $\mathrm{K}$ to $60 \mathrm{~K}$. The diffraction pattern changes at around $65(5) \mathrm{K}$ and all the samples are strongly damaged, as usually observed at the spin crossover for this compound, indicating the HS to LS relaxation at this temperature.

\section{Results and discussions}

\subsection{Unit cell along the hysteresis loop}

The iron(II) mononuclear complex denoted $\left[\mathrm{Fe}(\mathrm{PM}-\mathrm{PeA})_{2}(\mathrm{NCS})_{2}\right]$ is known to exhibit a thermal induced spin crossover with a large hysteresis. Magnetic measurements gives a temperature of transition of $\mathrm{T} 1 / 2 \downarrow=188 \mathrm{~K}$ when cooling and $\mathrm{T} 1 / 2 \uparrow=238 \mathrm{~K}$ when warming [10] showing a hysteresis of $40 \mathrm{~K}$. Previous X-ray structural investigation indicated a strong re-arrangement at the spin crossover from a monoclinic P2 ${ }_{1} / \mathrm{c}$ unit cell in HS to an orthorhombic Pcen unit cell in LS [16]. Up to now the unit cell temperature dependence along the hysteresis loop could not be determined due to the deterioration of the samples at the structural transition. This has been achieved here by single crystal X-ray diffraction (Figure 1). The determination of the unit cell parameters and volume variations from 280 to $140 \mathrm{~K}$ in the warming and cooling modes confirms the structural transition and evidences an hysteresis width of $19 \mathrm{~K}$.

First, the temperatures of transition deduced from the structural data do not correspond to those obtained by magnetic measurements, especially in the cooling mode: T1/2 $\downarrow=215 \mathrm{~K}$ when cooling and $\mathrm{T} 1 / 2 \uparrow=234 \mathrm{~K}$. The small difference for the latter $(4 \mathrm{~K})$ can easily be explained taking into account experimental errors but the former shows a significant difference of $17 \mathrm{~K}$ not explained today. Both magnetic and X-ray measurements are reproducible. One can note however that magnetic measurements were performed on powder sample or on a large number of single crystals while X-ray experiments are performed on one single crystal only. No differences have been observed between the experimental powder diffractogram and the diffractogram simulated from the single-crystal structure atomic parameters. This indicates that crystallographic phases are identical. However, it is well known that spin crossover characteristics are very sensitive to crystal defects, especially solvent inclusion. The family of complexes of which $\left[\mathrm{Fe}(\mathrm{PM}-\mathrm{PeA})_{2}(\mathrm{NCS})_{2}\right]$ belonged is indeed known to be potentially affected by solvent inclusion [16]. If these defects affect randomly the samples then magnetic measurements based on the use of a large amount of crystallite are more likely affected. The presence of defects is thus probably at the origin of the above differences. Consequently, true temperatures of 
spin crossover are those given by single crystal measurements. This assumption might be generalized to all spin crossover complexes.

The amplitude of the volume variation of the sample at the macroscopic level is an interesting feature as it could be used in a large number of potential applications, such as molecular motors for instance [17]. The macroscopic modification of the crystal follows the modification of the unit cell. The unit cell temperature dependence shows that the spin transition corresponds to a reversible modification of the unit cell volume of $4.2 \%$, which is by far the largest change observed for a mononuclear complex (Figure 1a). But one of the crucial point to enhanced is the anisotropy of the unit cell modification (Figure $1 \mathrm{~b})$. The $b$ parameter is not affected by the transition $(-0.2 \%)$ while the c parameter increases $(+4.4 \%)$ and the $a$ parameter strongly decreases $(-8.6 \%)$ from HS to LS. Amplitudes of the modification are strictly the same from LS to HS but obviously in the opposite senses. These unit cell modifications correspond by definition to the macroscopic sample modifications at the spin-crossover that consequently are strongly anisotropic. This feature appears almost frequent among spin crossover complexes [18] even though unusually pronounced in the present case. As a general matter of fact, the anisotropy of the contraction-dilatation process at the spin crossover should therefore be taken into account in any attempt to use the sample volume variation in potential application.

\subsection{Simultaneity of structural and spin transitions}

Concerning the mechanism of spin crossover, knowing which of the structural transition and the spin crossover drives the other one or vice versa often corresponds to an "egg and chicken" problem. In the present case, the occurrence of a large structural re-organisation together with the spin crossover corresponding to a change of unit cell symmetry, from monoclinic $\mathrm{P} 22_{1} / \mathrm{c}$ to orthorhombic Pccn, deserves to re-investigate this question. We tried to differentiate these phenomena by investigating the quenching effect. A single crystal was rapidly cooled from $293 \mathrm{~K}$ to $30 \mathrm{~K}$ and the crystal structure determined. The monoclinic-orthorhombic transition is quenched. The $30 \mathrm{~K}$ unit cell is very close from the high temperature HS one. The crystal packing is identical to the already known HS crystal structure at room temperature [10]. The iron(II) coordination sphere geometry also clearly indicates that the complexes are in the HS state, even though a small LS residue cannot be excluded, as shown by the metal-ligand bond lengths $(<\mathrm{Fe}-\mathrm{N}>=2.107(1) \AA)$ and the distortion parameters $\left(\Sigma=73.7(1)^{\circ}\right)$ in agreement with the HS values for this kind of complexes [18]. This result evidences in the present case the synergy between the structural transition and the spin conversion, showing that they cannot be dissociated. This is enhanced by the unit cell parameter temperature dependence by warming after a quench at $30 \mathrm{~K}$ (Figure 2). Unsurprisingly, the unit cell volume relaxes towards the unit cell volume expected after a slow cooling. It has been indeed previously demonstrated, from magnetic and structural measurements that quenched HS states relax towards LS state after warming [19]. The deterioration of the single crystals along this process is clearly on line with the structural transition, 
known to be destructive for the single crystal in this sample. Thus, spin conversion and structural transition cannot be differentiated in this sample and must probably not be seen as separated phenomena.

This result also indicates that, contrary to what was initially believed, the temperature beyond which a photo-conversion is efficient, namely T(LIESST), is not necessary the same that the temperature corresponding to the HS to LS conversion after quenching, namely T(TIESST). For the studied complex, the former is around $30 \mathrm{~K}$ [20] while the present study shows the latter is around $65(5) \mathrm{K}$.

\subsection{Spin-like domains temperature dependence}

The single crystal investigation unambiguously characterizes a spin-like domains distribution, referring to process (2) described in the above introduction, as shown by the reversible splitting of the Bragg peaks in the spin crossover zone (Figure 3). However the alteration of the single crystals around this temperature urged us to perform the temperature dependence of the spin domains on powder samples in order to obtain reliable quantitative data. Analysis of the powder diffractograms clearly confirms the spin-like domains mechanism of the transition for this compound (Figure 4). This is especially clear on the more intense Bragg peak [110] on which we can focus on. As expected for spin domains, in the cooling mode the LS component appears around $205 \mathrm{~K}$, when the intensity of the HS component starts to decrease, and is unique at around $165 \mathrm{~K}$ when the HS component is not anymore observable. Then, in the warming mode, the HS component reappears at around $215 \mathrm{~K}$ to be unique at around $235 \mathrm{~K}$. The hysteresis loop is consequently perfectly described by the spin domains formation process.

The [110] Bragg peak intensity temperature dependence (Figure 5) first shows that the spin conversion region corresponds to a co-existence of the HS and LS spin domains on a large temperature range of about $40 \mathrm{~K}$ in the cooling mode and $20 \mathrm{~K}$ in the warming mode. Consequently, the transition is not as abrupt as expected, especially on the cooling mode. Note that these results are strictly reproducible either on subsequent complete thermal cycles or on other samples.

The temperatures for which the intensities of the HS and LS [110] Bragg peaks are identical give the $T_{1 / 2}$ values in the corresponding mode, $T_{1 / 2 \downarrow}=190 \mathrm{~K}$ and $T_{1 / 2 \uparrow}=232 \mathrm{~K}$. The hysteresis width appears thus of around $40 \mathrm{~K}$. These values are on line with those deduced from the magnetic measurements that are also performed on powder or/and on a large number of single crystals but is half smaller than the hysteresis width obtained from the above X-ray investigation on one single crystal, i.e. $20 \mathrm{~K}$. As already noticed above, the latter can certainly be considered as the true structural hysteresis width. Indeed, measurements performed on powder or equivalent sample probably mirror some additional inhomogeneity within the grains. This is for example reflected by the [110] intensity temperature dependence curves that present an assymetric shape nearby $\mathrm{T}_{1 / 2}$, the low temperature parts 
modifications being smoother than the high temperature ones. The nature of this inhomogeneity is to be determined but may find its origin into crystal defects.

Elsewhere, it has been recently demonstrated from spectroscopic measurements on other iron spin-crossover complexes that it is possible in certain conditions to induce a photo-conversion within the hysteresis loop using a pulsed laser beam at room temperature [21]. These promising results question however the stability of the structure of the sample at the microscopic and macroscopic levels within the hysteresis loop. This stability appears crucial for the non-fatigability and the reproducibility of the conversion within the hysteresis loop. The iron(II) compounds then studied, for which no single crystals were available, were however known to be easily photo-switchable. In the case of [Fe(PM$\mathrm{PeA})_{2}(\mathrm{NCS})_{2}$ ] the core of the loop cannot be accessed by irradiation effects but it is possible to access it by temperature effects instead. Even though no spin crossover is expected by thermal effects in the hysteresis loop, X-ray investigation can gives preliminary information on the thermal stability of the sample in this loop. The sample was thus first cooled down to $T_{1 / 2 \downarrow}$. and the Bragg peaks shapes were then registered every $5 \mathrm{~K}$ from $T_{1 / 2 \downarrow}$ to $T_{1 / 2 \uparrow}$ from powder diffractrograms. This allows to follow the spin-like domain HS to LS ratio temperature dependence (Figure 6). First the shape of the corresponding HS and LS Bragg peaks remains unchanged in all the hysteresis loop testifying no modifications of these domains. This result also proves the samples are not damaged by such warming at any scale level. The intensities of the Bragg peaks are not modified during the warming up to $T_{1 / 2 \uparrow}$. This indicates that the HS and LS domains ratio (0.5) is not affected by such warming. Consequently, this spin like domain temperature dependence from $T_{1 / 2 \downarrow}$ to $T_{1 / 2 \uparrow}$ demonstrates the thermal stability of the sample at the microscopic and the macroscopic levels inside the magnetic hysteresis loop.

\section{Conclusions}

The investigation of the $\left[\mathrm{Fe}(\mathrm{PM}-\mathrm{PeA})_{2}(\mathrm{NCS})_{2}\right]$ complex first confirms the assumption that one as to be very careful when comparing spin crossover features from experiments performed using powder and single crystals samples. In particular temperatures of transition can be very sensitive to the nature of the sample, probably because of the possible presence of crystal defects. Then, it has been shown that the very large and anisotropic unit cell modifications and the spin crossover phenomenon are probably indissociable in the present case. Elsewhere, X-ray powder diffraction has been evidenced as a powerful tool to visualize and investigate the mechanism of transition, screening here a spin-like domains process. Investigation of the hysteresis loop has revealed that it is possible to navigate inside the loop without damaging the sample and without modifying the spin-like domains shapes and ratio. One of the new routes to get photo-induced high temperature spin crossover devices aims to induce the conversion inside the hysteresis loop [21, 22]. To this regard the present results stating the microscopic and macroscopic thermal stability of the sample inside the loop represent an encouraging feature. Obviously, in order to go further on that point, X-ray investigation of a photo- 
conversion inside a hysteresis loop should now be attempted on a well selected compound, for example by mean of time resolved $\mathrm{X}$-ray diffraction.

\section{References}

[1] Eds. Gütlich P and Goodwin H A 2004 Spin crossover in Transition Metal Compounds, Topics in Current Chemistry, Springer-Verlag Berlin Heidelberg NewYork volumes I, II and III.

[2] Irler W Ritter G König E Goodwin H A Nelson SM 1979 Solid Stat. Comm. 2939

[3] Pillet S Hubsch J and Lecomte C 2004 Eur. J. Phys. B 38541

[4] Huby N Guérin L Collet E Toupet L Ameline J C Cailleau H Roisnel T Tagayaki T and Tanaka K 2004 Phys. Rev. B 69 020101(R)

[5] Ichiyanagi K Hebert J Toupet L Cailleau H Guionneau P Létard J -F and Collet E 2006 Phys Rev B 73 060408(R)

[6] Legrand V Carbonera C Pillet S Souhassou M Létard J F Guionneau P and Lecomte C 2005 J. Phys. : Conf. Ser. 2173

[7] Goujon A Gillon B Debede A Cousson A Gukasov A Jeftic J McIntyre G J and Varret F 2006 Phys. Rev. B 73104413

[8] Chernyshov D Hostettler M Tönroos K W and Bürgi H B 2003 Angew. Chem. Int. Ed. 423825

[9] Pillet S Lecomte C Sheu C F Lin Y C Hsu I J and Wang Y 2005 J. Phys.: Conf. Ser. 21221

[10] Létard J -F Guionneau P Codjovi E Olivier L Bravic G Chasseau D and Kahn O 1997 J. Am. Chem. Soc. 19910861

[11] Ballon J Comparat and V Pouxe 1983 J. Nucl. Instrum. Methods 217213

[12] Evain M Deniard P Jouanneaux A and Brec R 1993 J. Appl. Cryst. 26563

[13] Huang T C Toraya H Blanton T N and Wu Y 1993 J. Appl. Cryst 26180

[14] Programs for Crystal Structure Analysis (Release 97-2) Sheldrick GM 1998 Institüt für Anorganische Chemie der Universität, Tammanstrasse 4, D-3400 Göttingen, Germany

[15] Farrugia L J 1999 J. Appl. Cryst. 32837

[16] Guionneau P Létard J -F Yuffit D S Chasseau D Howard J A K Goeta A E and Kahn O 1999 J. Mater. Chem. 4985

[17] Létard J -F Guionneau P and Goux-Capes L 2004 Topics in current Chemistry 235221

[18] Guionneau P Marchivie M Bravic G Létard J -F and Chasseau D 2004 Topics in current Chemistry 23497

[19] Marchivie M Guionneau P Létard J -F Chasseau D and Howard J A K 2004 J. Phys. Chem. Sol. 6517

[20] Létard J -F Guionneau P, Nguyen O Costa J S Marcen S Chastanet G Marchivie M and Goux Capes L 2005 Chem. Eur. J. 114582 
[21] Bonhommeau S Molnar G Galet A Zwick A Real J A McGarvey J J Bousseksou A 2005 Angew. Chem. Int. Ed. 444069

[22] Freysz E Montant S Létard S Létard J -F 2004 Chem. Phys. Lett. 394318 
Figure 1. Relative unit cell temperature dependence along the hysteresis loop: (a) unit cell volume (b) unit cell parameters $a$ (square), $b$ (circle) and $c$ (triangle) determined by mean of single crystals X-ray diffraction. References are the $280 \mathrm{~K}$ unit cell parameters $\mathrm{a}=15.669(1) \AA \hat{,} \mathrm{b}=14.535(1) \AA$, $\mathrm{c}=16.865(2) \AA \hat{,}, \beta=93.18(1)^{\circ}, \mathrm{V}=3834(1) \AA^{3}$. Molecular scheme of the complex is inserted.

Figure 2. Temperature dependence of the unit cell volume after a quench at $30 \mathrm{~K}$ (square) or a slow cooling (triangle) . Single crystals are seriously damaged during the HS to LS relaxation

Figure 3. Single crystal X-ray diffraction pattern showing the splitting of a Bragg peak in the conversion temperature region.

Figure 4. (a) Selected X-ray powder diffractograms along the hysteresis loop from down to up and (b) zoom on the [110] Bragg peak showing the reversible splitting of the peaks.

Figure 5. Detailed temperature dependence of the intensity of the [110] Bragg peaks corresponding to the HS (square) and the LS (circle) domains (a) in the cooling mode and (b) in the warming mode.

Figure 6. Temperature dependence of the intensity and of the shape of the [110] Bragg peaks corresponding to the HS and the LS domains inside the hysteresis loop. 
FIGURE 1

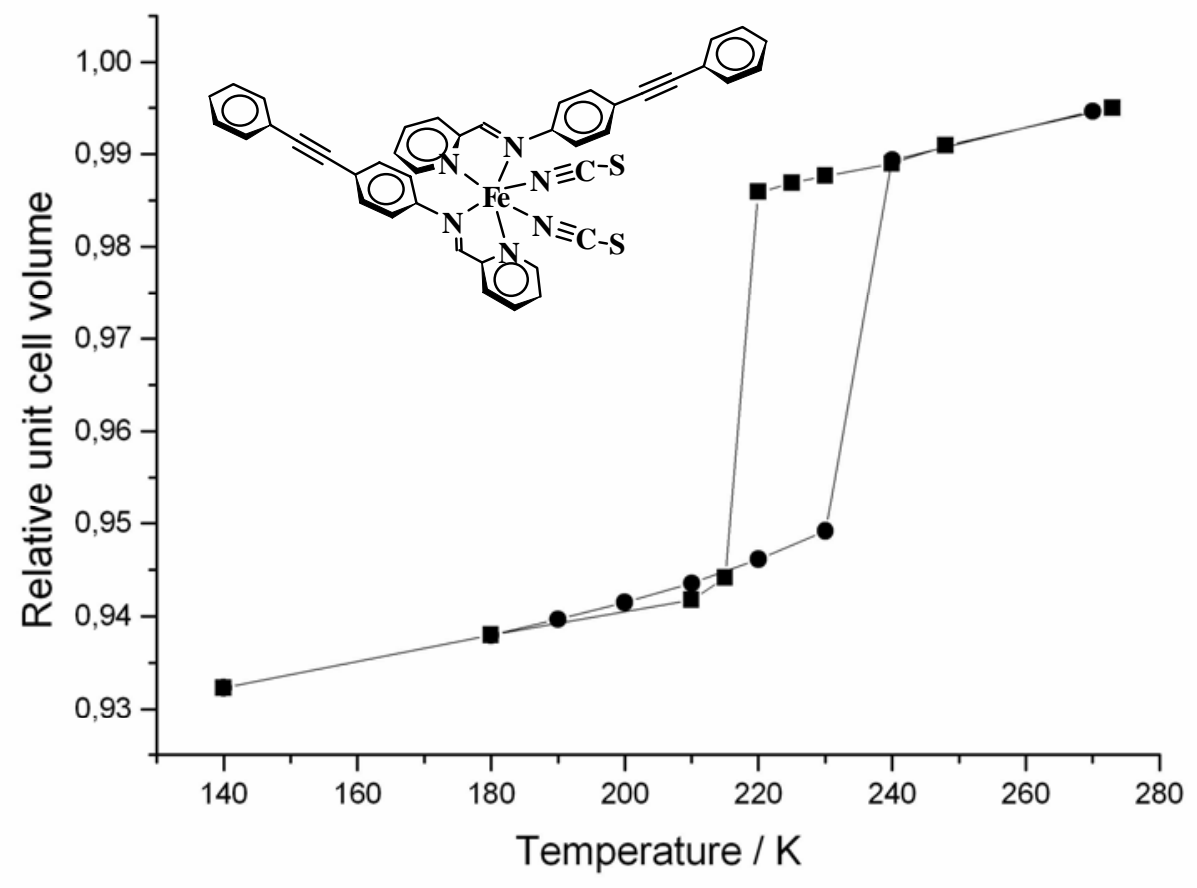

(a)

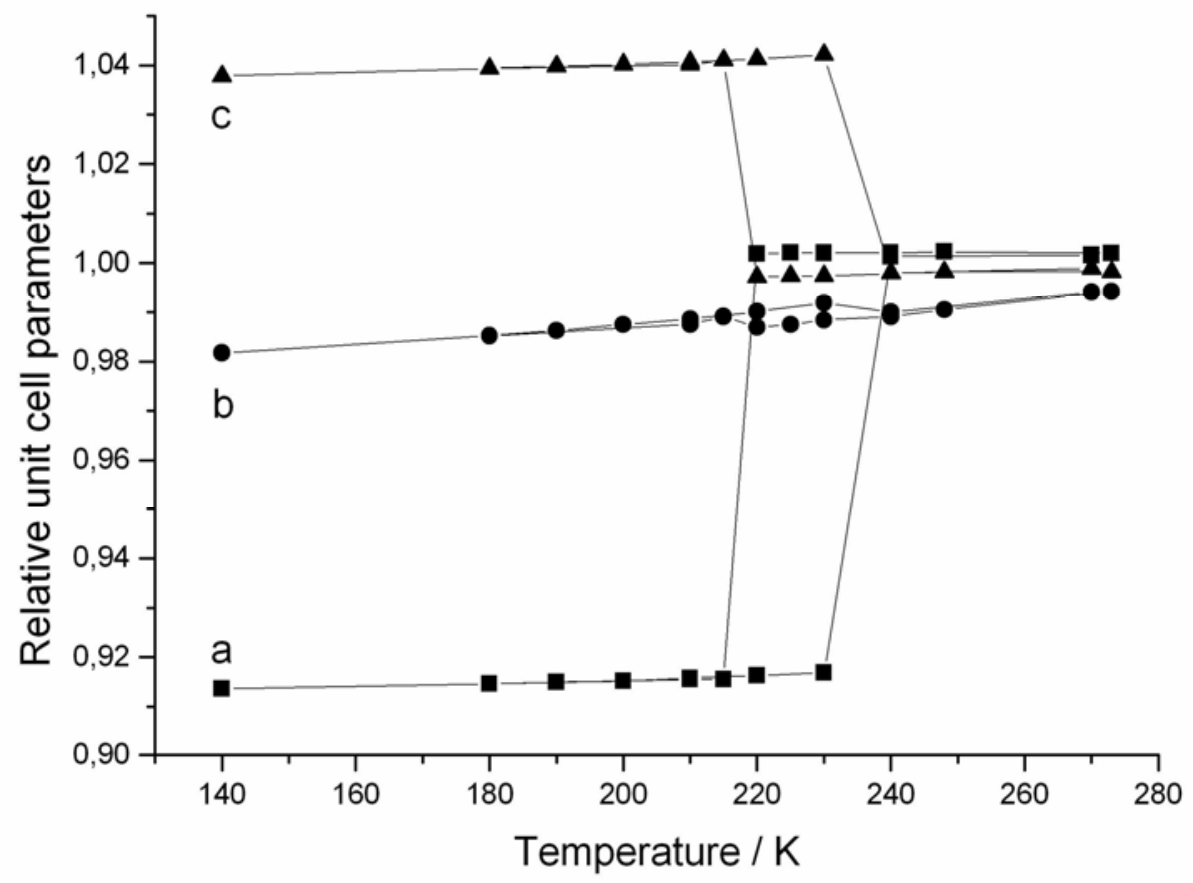

(b) 


\section{FIGURE 2}

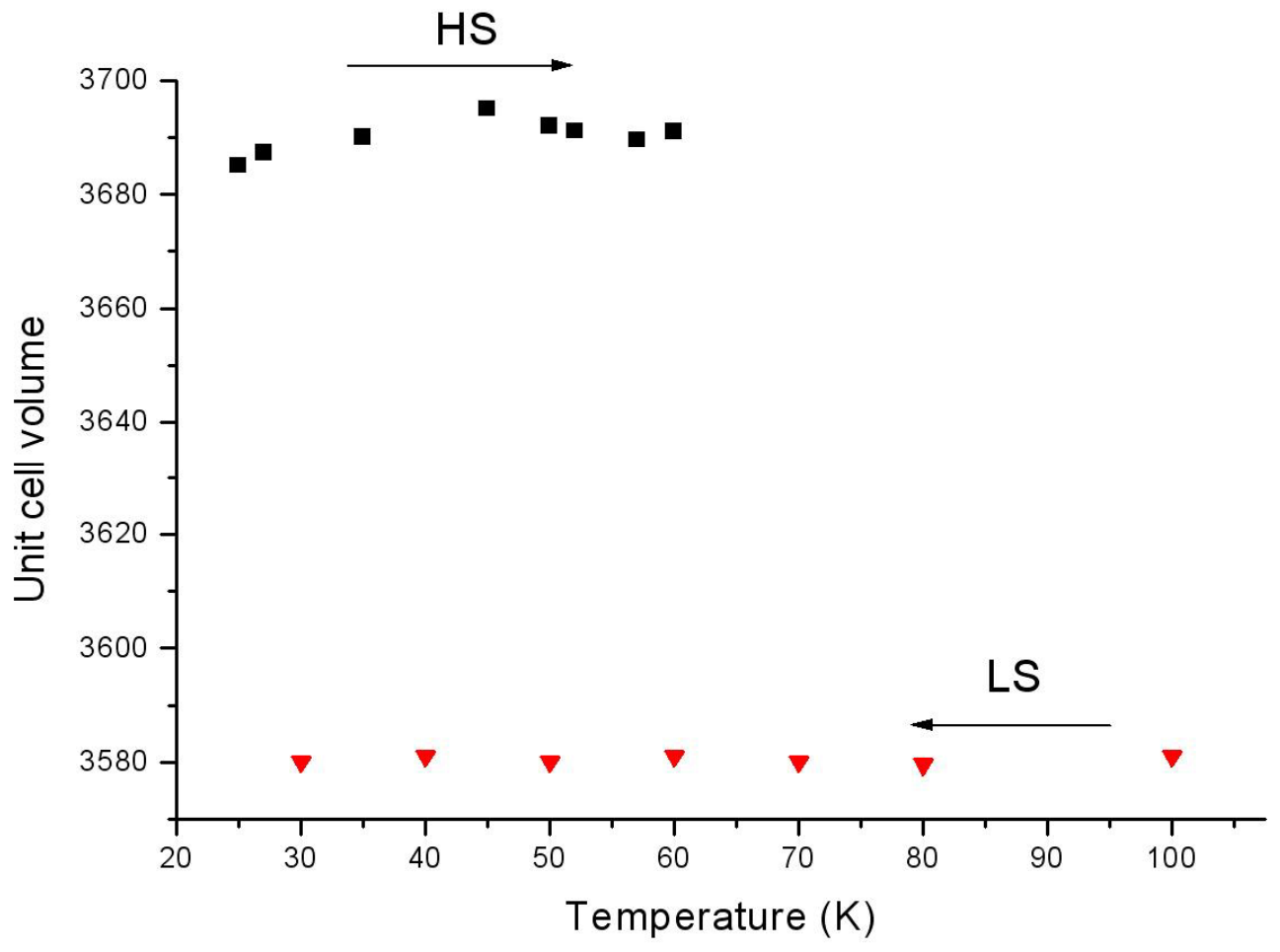




\section{FIGURE 3}

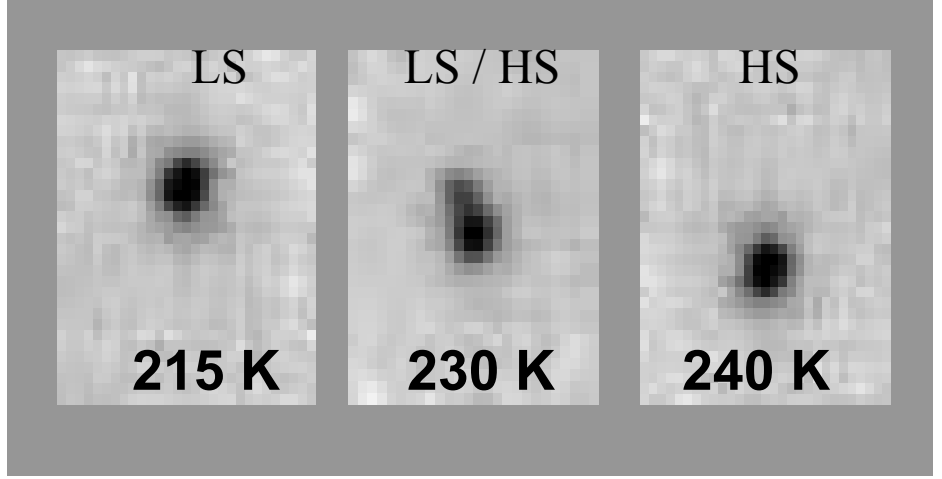


FIGURE 4

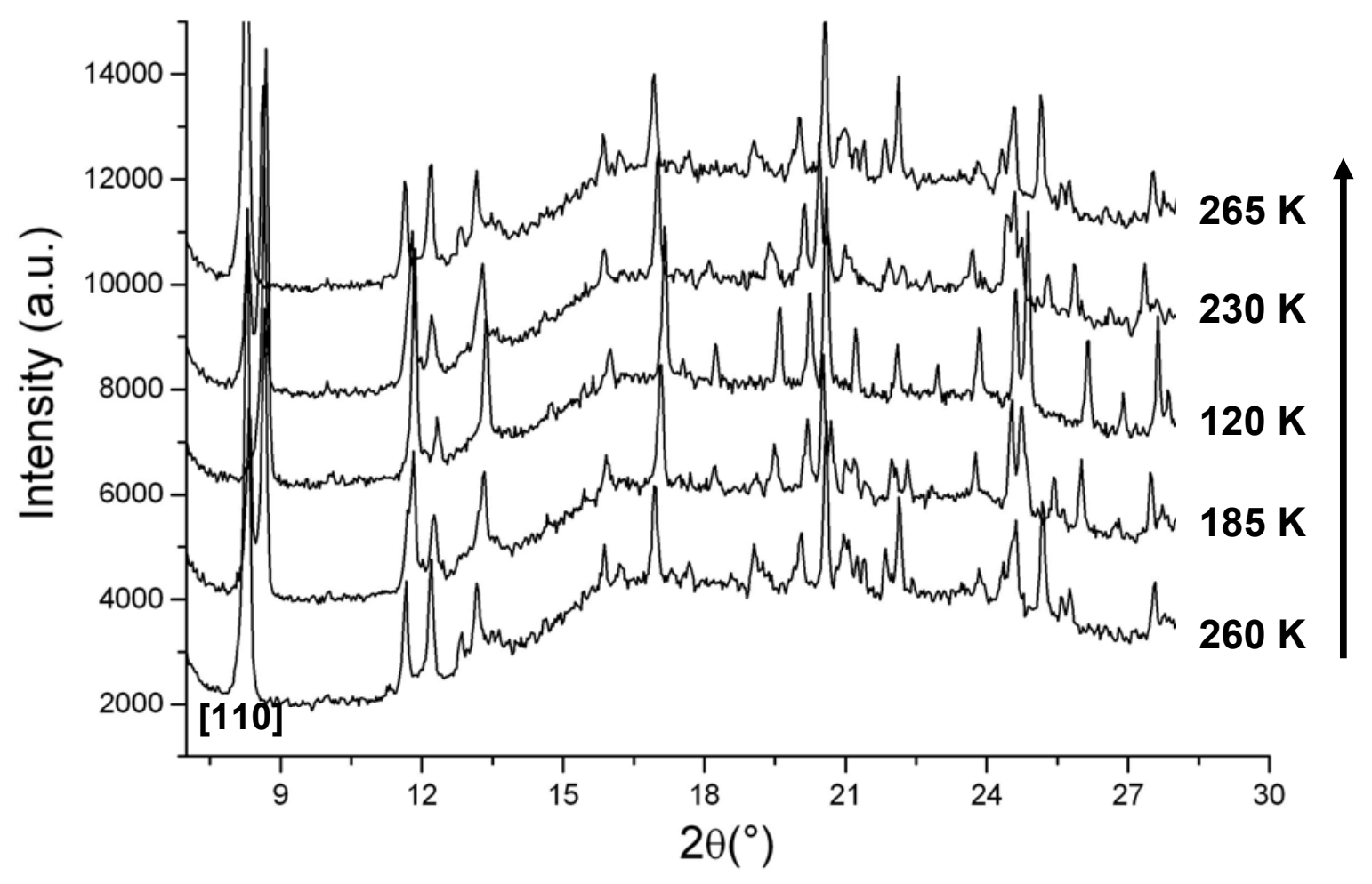

a)

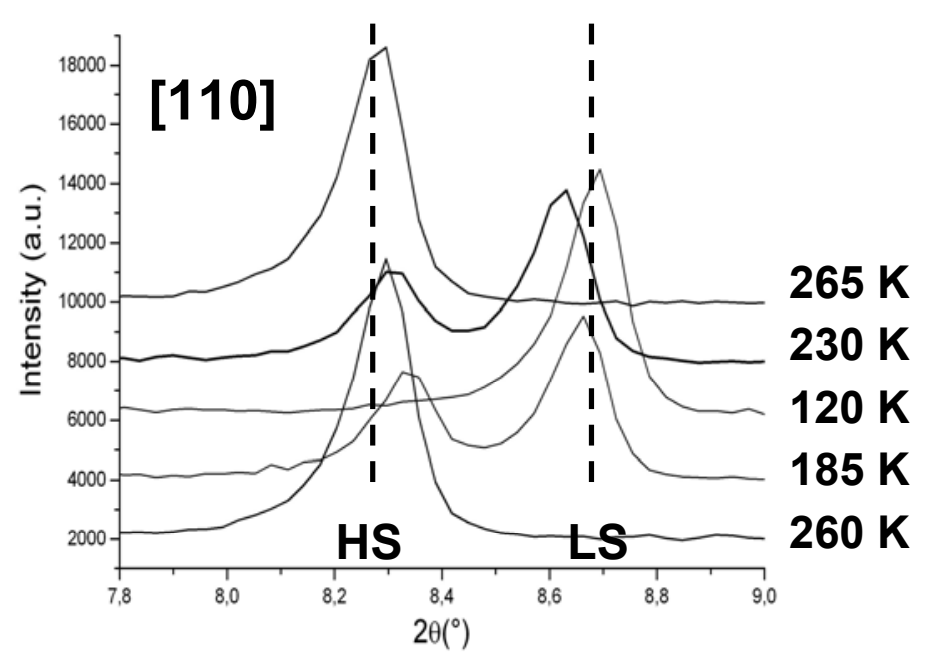

b) 
FIGURE 5

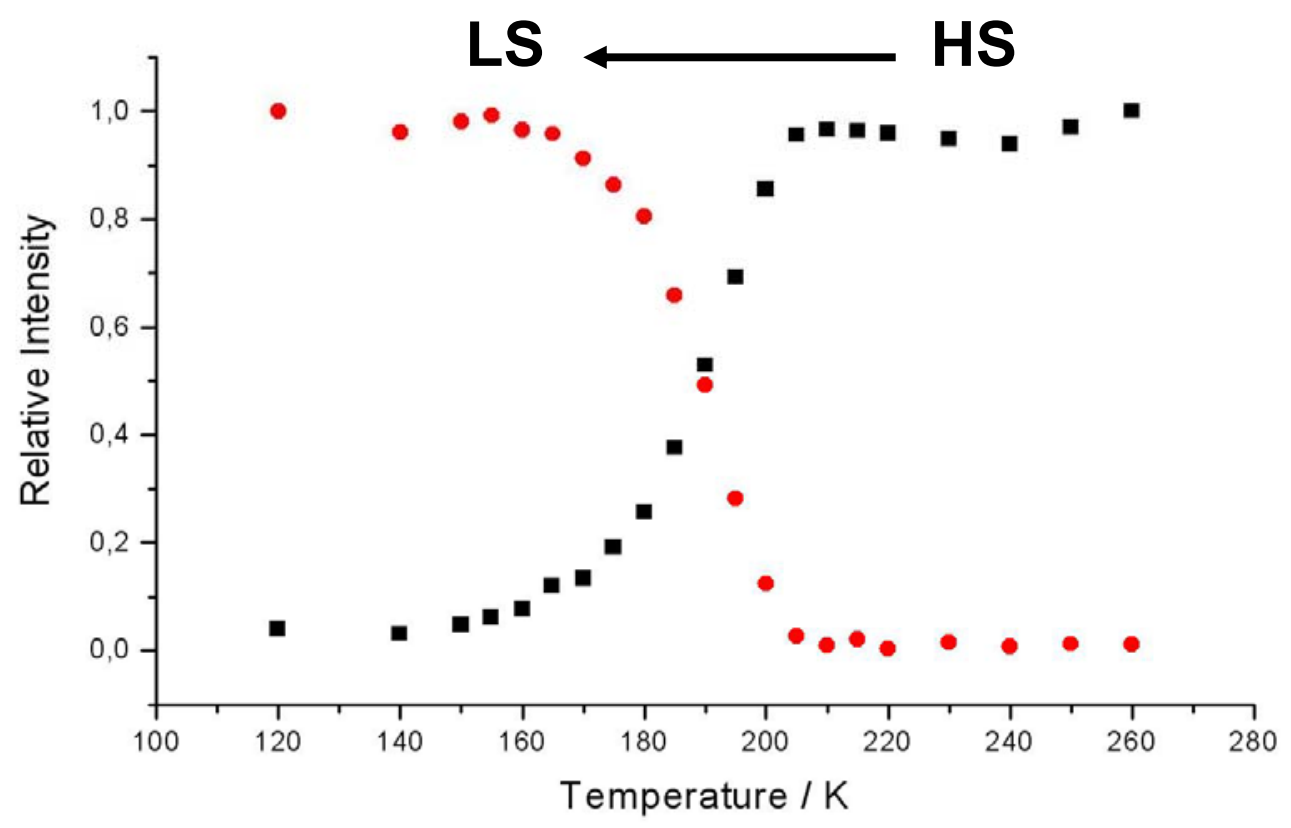

a)

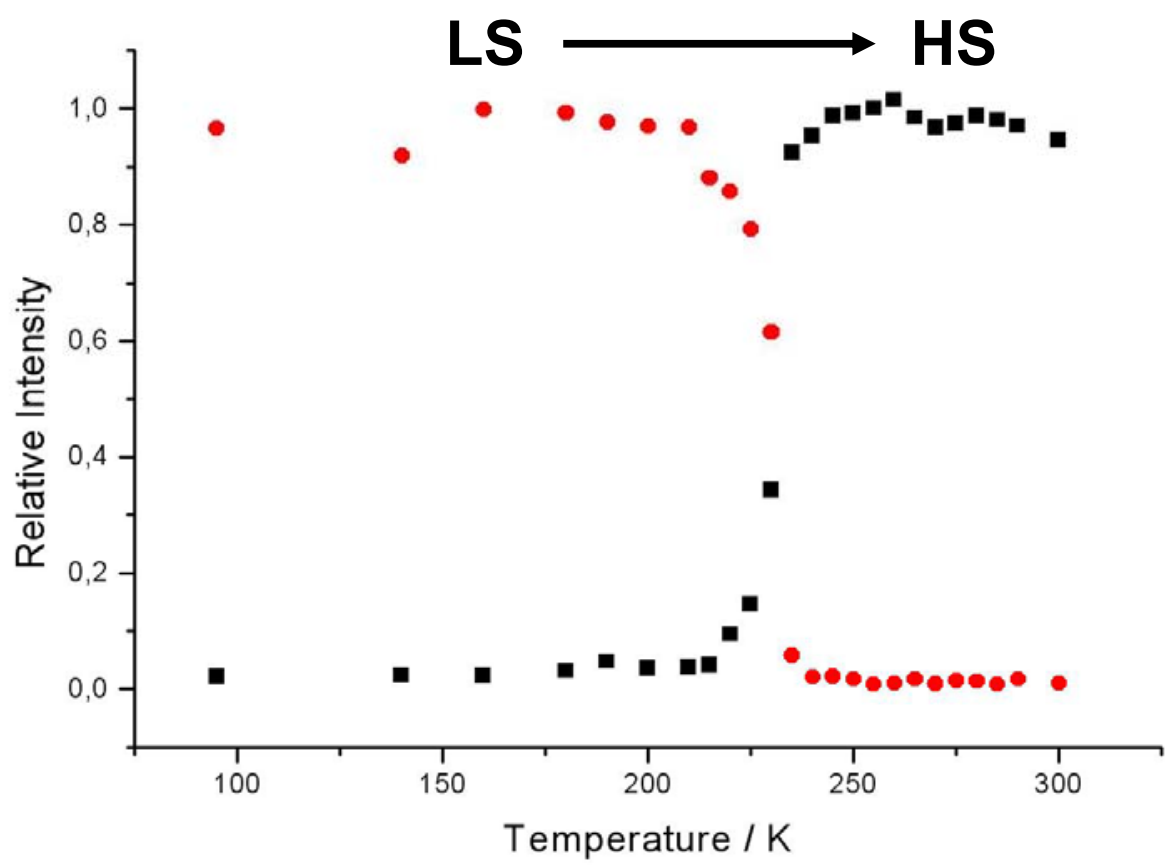

b) 
FIGURE 6

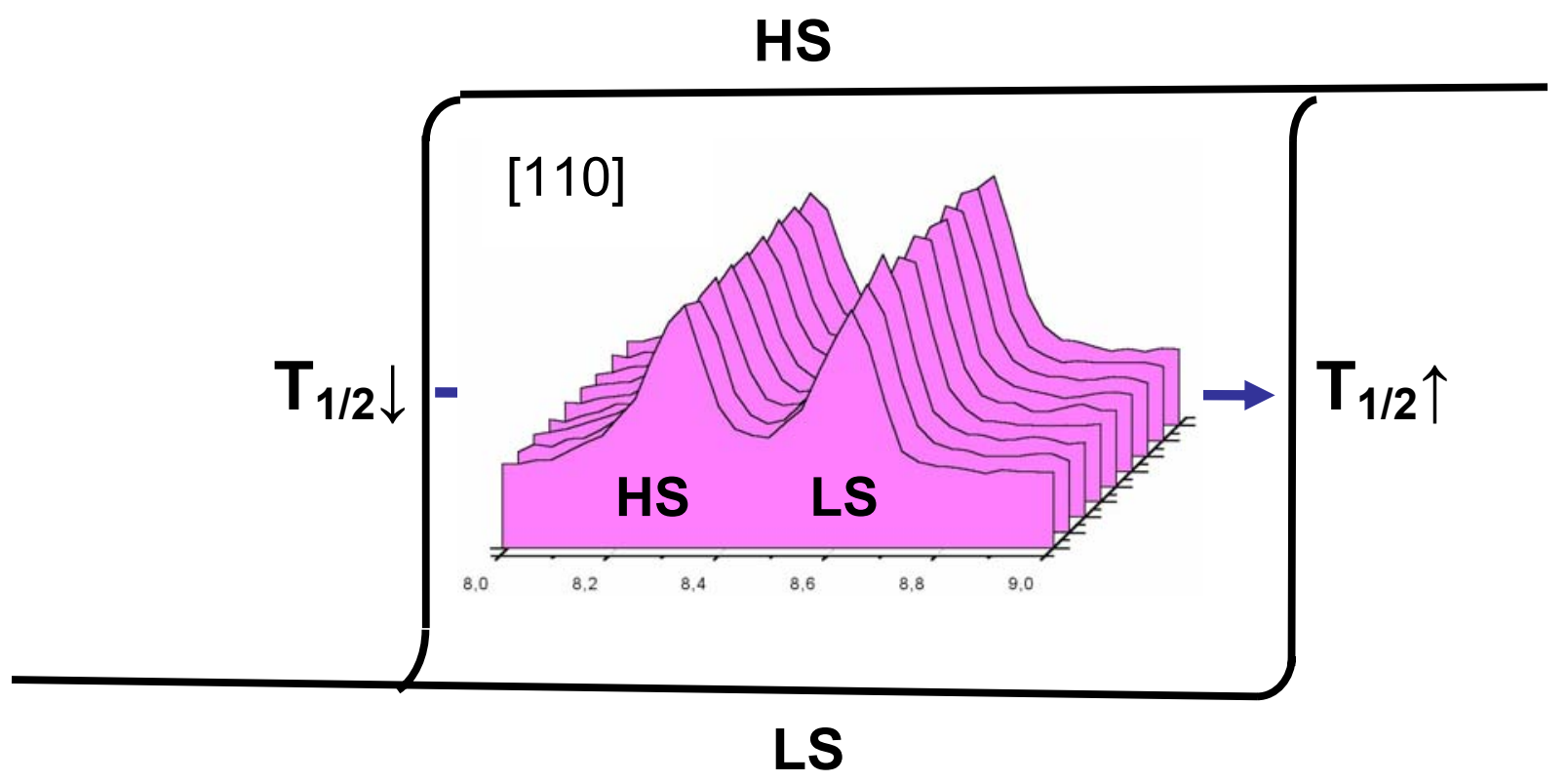

